\title{
Dihydromyricetin ameliorates transverse aortic constriction induced myocardial hypertrophy in mice via inhibition of oxidative stress
}

\author{
Yun Chen, Guoliang Meng, Wei Zhang
}

School of Pharmacy, Nantong University, China

Background Dihydromyricetin (DMY), one of the flavonids in vine tea, exerts many pharmacological actions, such as hypoglycemic, antihypertensive, and anti-oxidative effects. However, it is not clear whether DMY has a protective effect on pressure overload induced myocardial hypertrophy.

Methods Transverse aortic constriction was performed in male C57BL/6 mice aging 8-10 weeks after 2 weeks of DMY intragastric administration. DMY was given for another 2 weeks after TAC. Blood pressure and cardiac index were observed. Hematoxylin-eosin (HE) staining was used to detect the cardiomyocyte cross-sectional areas. Expression of arterial natriuretic peptide (ANP), brain natriuretic peptide (BNP), sirtuin 3 (SIRT3) and forkhead-box-protein 3a (FOXO3a) were detected with real-time PCR or/and western blot. The level of oxidative stress was assessed with dihydroethidium (DHE) staining in myocardial tissue. In addition, the level of malondialdehyde (MDA), activity of superoxide dismutase (SOD) and total antioxidant capacity (T-AOC) were evaluated.

Results There was no significant difference on blood pressure after DMY pretreatment. DMY significantly reduced cardiomyocyte cross-sectional areas and cardiac indexes in mice after TAC. In addition, DMY pretreatment significantly decreased ANP, BNP expressions at both mRNA and protein level, but significantly increased the expression of SIRT3, FOXO3a and SOD2. DMY also attenuated reactive oxygen species production and MDA level, while enhanced the SOD activity and T-AOC after TAC.

Conclusions Dihydromyricetin ameliorated TAC induced myocardial hypertrophy in mice, which might be related to the inhibition of oxidative stress. 